\title{
Investigation of the Static Nuclear Properties of Some Cr-Isotopes Using Skyrme-Hartree-Fock Method
}

\author{
Ehsan M. Raheem ${ }^{*}$ and Ali A. Abdul Hasan \\ Ministry of Science and Technology, Directorate of Nuclear Researches and Applications, \\ Baghdad- Iraq. \\ *Corresponding Author: ehsan.nucl@yahoo.com.
}

\begin{abstract}
The self-consistent Hartree-Fock (HF) calculations with several Skyrme parameterizations; SKM, SKM*, SLY4, SKxs25, MSK7, SKO, SKxta, and SKxtb have been used to investigate some static nuclear ground-state properties of some Chromium $\left({ }^{50-54} \mathrm{Cr}\right)$ isotopes. The nuclear ground state charge, mass, neutron and proton densities with the corresponding root mean square radii, the charge form factors, the total binding energies and the neutron skin thickness can be calculated. The calculations are performed using NuShellX@MSU code, where the deduced results led to specifying one set or more of Skyrme parameterizations that used to achieve the best agreement with the available experimental data. [DOI: 10.22401/ANJS.22.2.05]
\end{abstract}

Keywords: Nuclear ground-state properties, Skyrme interaction, Skyrme-Hartree-Fock method, nuclear density, Chromium isotopes.

\section{Introduction}

Nuclear structure theory aims to build an extensive microscopic framework in which the nuclear properties can be explained. Many microscopic theoretical methods were focused on studying the nuclear structure of nuclei that are located near and far from the stability line. The Hartree-Fock (HF) self-consistent meanfield method in addition to several effective interactions provide an appropriate description of the nuclear structure of nuclei and the ground state properties such as binding energies and nuclear density distributions with their corresponding root mean square (rms) radii.

A considerable simplification of $\mathrm{HF}$ calculations was achieved with the aid of Skyrme effective interaction which is considered as the most common interaction. The combination between HF method and Skyrme interaction forms an effective model named Skyrme-Hartree-Fock (SHF) model, which has the ability to explain successfully various nuclear properties such as density distributions, nuclear radii and nuclear deformations $[1,2]$.

The nuclear ground state properties, especially the nuclear density distributions, give much detailed information about the shape and size of nuclei since they are related to the wave functions of nucleons and represent as very important key points for many nuclear studies and investigations. Furthermore, these properties offer a precise test for many nuclear models and provide valuable and accurate details concerning the nuclear structure.

The high-energy electron scattering experiments from nuclei provide clear conceptions about the nuclear charge density distribution (CDD) and the related charge form factor, where both nuclear properties carry exactly the same amount of information [3].

The aim of this research is to investigate several static ground state properties of ${ }^{50-54} \mathrm{Cr}$ isotopes using the SHF method with Skyrme parameterizations; SKM [4], SKM* [4], SLY4 [5], SKxs25 [6], MSK7 [7], SKO [8], SKxta [9], and SKxtb [9]. The calculations are performed using NuShellX@MSU code [10] and the deduced results will be compared with the available experimental data.

\section{Theory}

The Skyrme force is considered as the most appropriate force that can be used to describe the nuclear ground state properties in conjunction with HF calculations. This force consists of two parts; the first part $v_{i j}^{(2)}$ is the momentum dependent two-body part, and the second part $v_{i j k}^{(3)}$ is the zero-range three-body part. The Skyrme force is given by $[1,11]$ : 
$\widehat{V}_{\text {Skyrme }}=\sum_{i<j} v_{i j}^{(2)}+\sum_{i<j<k} v_{i j k}^{(3)}$

with:

$v_{i j}^{(2)}=t_{0}\left(1+x_{0} P_{\sigma}\right) \delta_{i j}$

$+\frac{1}{2} t_{1}\left[\delta_{i j} \hat{\vec{k}}^{2}+\hat{\vec{k}}^{\prime 2} \delta_{i j}\right]$

$+t_{2} \hat{\vec{k}}^{\prime 2} \delta_{i j} \hat{\vec{k}}+i t_{4}\left(\hat{\vec{\sigma}}_{i}+\hat{\vec{\sigma}}_{j}\right) \cdot \hat{\vec{k}}^{\prime} \delta_{i j} \hat{\vec{k}}$

$v_{i j k}^{(3)}=t_{3} \delta_{i j} \delta_{j k}$

For even-even spin saturated nuclei, we have:

$v_{i j k}^{(3)} \approx v_{i j}^{(2)}=\frac{1}{6} t_{3}\left(1+P_{\sigma}\right) \rho_{0}^{\alpha}(R) \delta_{i j}$.

with:

$\hat{P}_{\sigma}=\frac{1}{2}\left(1+\hat{\vec{\sigma}}_{i} \cdot \hat{\vec{\sigma}}_{j}\right)$

where $\delta_{i j}=\left(r_{i}-r_{j}\right), \delta_{j k}=\left(r_{j}-r_{k}\right)$ are Dirac delta functions, $\hat{\vec{\sigma}}$ are the Pauli spin matrices, $P_{\sigma}$ is spin-exchange operator and $\rho_{0}(R)$ is the isoscalar density. The vectors $\widehat{\vec{k}}$ and $\hat{\vec{k}}^{\prime}$ are the relative (momentum) wave vector operators of two nucleons acting to the right and to the left, respectively; and given by:

$\hat{\vec{k}}=\frac{1}{2 i}\left(\vec{\nabla}_{i}-\vec{\nabla}_{j}\right), \hat{\vec{k}}^{\prime}=-\frac{1}{2 i}\left(\vec{\nabla}_{i}-\vec{\nabla}_{j}\right)$

The density dependent Skyrme interaction has the form $[12,13]$ :

$$
\begin{aligned}
& \widehat{V}_{\text {Skyrme }}(R, r)=t_{0}\left(1+x_{0} \hat{P}_{\sigma}\right) \delta(r) \\
& \quad+\frac{1}{6} t_{3}\left(1+x_{3} \hat{P}_{\sigma}\right) \rho_{0}^{\alpha}(R) \delta(r) \\
& \quad+\frac{1}{2} t_{1}\left(1+x_{1} \hat{P}_{\sigma}\right)\left[\hat{\vec{k}}^{\prime 2} \delta(r)+\delta(r) \hat{\vec{k}}^{2}\right] \\
& \quad+t_{2}\left(1+x_{2} \hat{P}_{\sigma}\right) \hat{\vec{k}}^{\prime} \cdot \delta(r) \hat{\vec{k}} \\
& \quad+i W_{0}\left(\hat{\vec{\sigma}}_{i}+\hat{\vec{\sigma}}_{j}\right) \cdot \hat{\vec{k}}^{\prime} \delta(r) \hat{\vec{k}}
\end{aligned}
$$

where $R=\left(r_{i}+r_{j}\right) / 2$ and $r=r_{i}-r_{j}$ are the center of mass coordinates and the relative distance, respectively. $t_{0}, t_{1}, t_{2}, t_{3}, W_{0}, x_{0}, x_{1}, x_{2}$, $x_{3}$ and $\alpha$ are the Skyrme free parameters describing the strengths of different interaction terms. These parameters are determined from the comparison between the calculated and the experimental nuclear ground state properties such as binding energies, nucleon densities and rms radii.
The charge, neutron and proton densities in spherical representation are given by [12]:

$\rho_{k}(r)=\sum_{\beta \in k} w_{\beta} \varphi_{\beta}(r)^{*} \varphi_{\beta}(r), k=c h, n, p$

where $w_{\beta}$ and $\varphi_{\beta}$ are the occupation probabilities of the state $\beta$; and $k$ denote to the charge, neutrons and protons of the nucleus.

The neutron, proton and charge mean square radii are given by [14]:

$\left\langle r^{2}\right\rangle_{k}^{1 / 2}=\left[\frac{\int_{0}^{\infty} d r r^{2} \rho_{k}(r)}{\int_{0}^{\infty} d r \rho_{k}(r)}\right]^{1 / 2}$

The neutron skin thickness is a very important quantity utilized to describe the distributions of neutrons in nuclei. It is defined as the difference between the neutron and the proton rms radii as [15]:

$$
\begin{aligned}
t=\Delta r_{n p} & =r_{n}-r_{p} \\
& =\left\langle r^{2}\right\rangle_{n}^{1 / 2}-\left\langle r^{2}\right\rangle_{p}^{1 / 2}
\end{aligned}
$$

The charge form factors are obtained from the ground-state CDDs, where the form factor is simply transformed to the CDD with using the Fourier-Bessel transformations and vice versa $[16,17]$. Therefore:

$F_{c h}(q)=\frac{4 \pi}{Z} \int_{0}^{\infty} \rho_{c h}(r) j_{0}(q r) r^{2} d r$

where $j_{0}(q r)=\sin (q r) / q r$ is the zeroth order spherical Bessel function and $q$ is the momentum transfer from the incident electron to the target nucleus. In the limit $q \rightarrow 0$, the target will be represented as a point particle, and the form factor of this target is equal to unity $F(q \rightarrow 0)=1$.

\section{Results and Discussion}

Most nuclear ground state features, such as nuclear shapes and radii, for some Cr-isotopes, have been studied within this work with the aid of the HF method in addition to several common Skyrme parameterizations that are tabulated in Table (1). The calculations included the density distributions of nuclear charge, proton, neutron and mass as well as the associated radii. The total nuclear binding energies, the neutron skin thickness and the charge form factors have been investigated and compared with the available experimental results. 
The charge rms radii for the considered isotopes are calculated and listed in Table (2) and plotted in Fig.(1) together with the available experimental values [18]. The calculated charge rms values were found to increase progressively from ${ }^{50} \mathrm{Cr}$ to ${ }^{54} \mathrm{Cr}$ (from $\mathrm{N}=26$ to $\mathrm{N}=31$ ) as the neutron number increases. The calculated charge rms values for ${ }^{50} \mathrm{Cr},{ }^{52} \mathrm{Cr},{ }^{35} \mathrm{Cr}$ and ${ }^{54} \mathrm{Cr}$ with using SLY4, $\mathrm{SKM}^{*}$, SKxta, and SLY4 parameterizations, respectively; are more close to the experimental values than the other values that were deduced with using the other Skyrme parameterizations.

Table (1)

The Skyrme parametrizations used in the present work.

\begin{tabular}{|c|c|c|c|c|c|c|c|c|c|c|}
\hline & $t_{0}$ & $t_{1}$ & $t_{2}$ & $t_{3}$ & $W_{0}$ & $X_{0}$ & $X_{1}$ & $X_{2}$ & $X_{3}$ & $\alpha$ \\
\hline SKM [4] & -2645.0 & 385.0 & -120.0 & 15595.0 & 130.0 & 0.09 & 0.0 & 0.0 & 0.0 & 0.167 \\
\hline 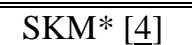 & $\overline{-2}$ & & & & & 0.09 & & 0.0 & 0.0 & \\
\hline & & $\overline{4}$ & & & 0 & 0.834 & $\overline{444}$ & $\begin{array}{l}-1.0 \\
\end{array}$ & 1.354 & \\
\hline$\overline{\text { SKx }}$ & $2-2887$ & 3 & & & 33 & .186 & & -0.601 & $\overline{0.409}$ & \\
\hline MSI & $\begin{array}{l}-182 \\
\end{array}$ & 259.400 & -292 & & 118.807 & 0.577 & -0.500 & -0.500 & 0.783 & 0.333 \\
\hline & & 303.4 & 791 & & $\begin{array}{l}118.0 \\
\end{array}$ & -0.211 & -2.81 & $\begin{array}{l}-1.46 \\
\end{array}$ & $\begin{array}{l}-0.43 \\
\end{array}$ & 0.25 \\
\hline & -1443.180 & 257.229 & -137.843 & 12139.420 & 180.441 & 0.341 & 0.580 & 0.167 & 0.0 & 0.500 \\
\hline SKxtb [9] & -1446.759 & 250.852 & -132.993 & 12127.649 & 153.054 & 0.329 & 0.518 & 0.139 & 0.018 & 0.500 \\
\hline
\end{tabular}

Table (2)

Calculated charge rms radii (fm) using different Skyrme parametrizations in comparison with experimental data.

\begin{tabular}{|c||c|c|c|c|c|c||c||c||c||}
\hline $\boldsymbol{A}$ & SKM & $\boldsymbol{S K M}^{*}$ & SLY4 & SKxs 25 & MSK7 & SKO & SKxta & SKxtb & EXP. [18] \\
\hline \hline 50 & 3.624 & 3.647 & 3.659 & 3.673 & 3.652 & 3.619 & 3.636 & 3.633 & $3.6588 \pm 0.0065$ \\
\hline \hline 52 & 3.623 & 3.646 & 3.659 & 3.668 & 3.656 & 3.619 & 3.642 & 3.638 & $3.6452 \pm 0.0042$ \\
\hline \hline 53 & 3.632 & 3.655 & 3.669 & 3.671 & 3.663 & 3.626 & 3.649 & 3.645 & $3.6511 \pm 0.0075$ \\
\hline 54 & 3.641 & 3.664 & 3.679 & 3.674 & 3.671 & 3.633 & 3.655 & 3.652 & $3.6885 \pm 0.0074$ \\
\hline
\end{tabular}

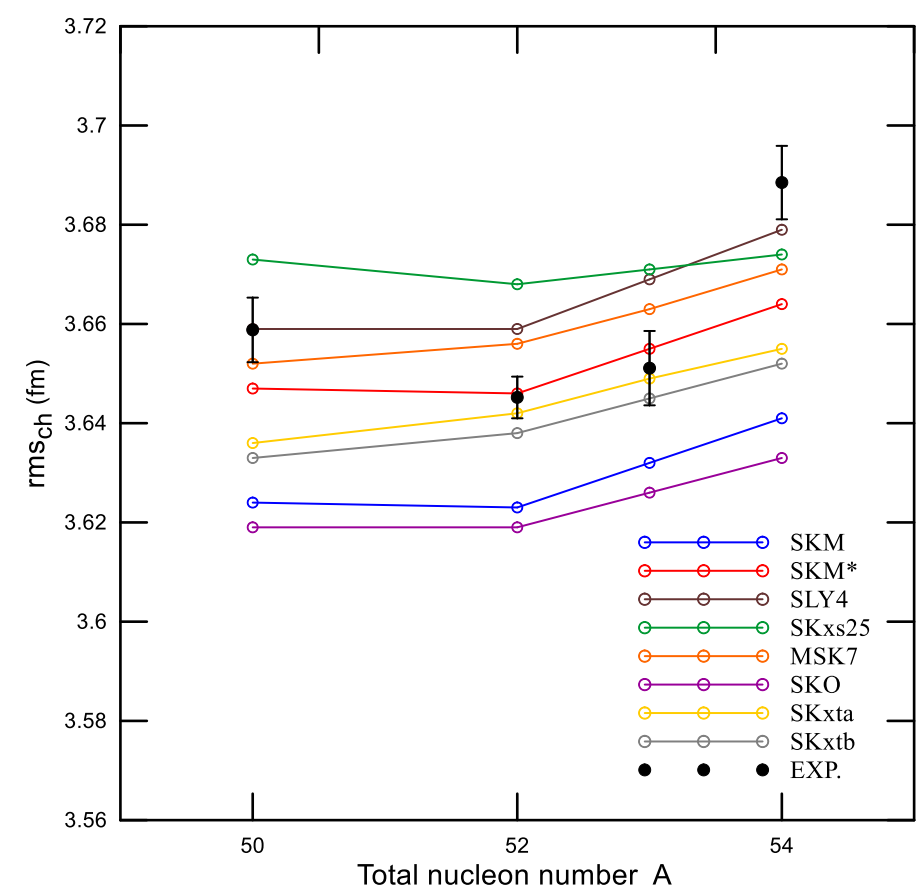

Fig.(1): Calculated rms charge radii for ${ }^{50-54}$ Cr isotopes compared with the experimental data taken from Ref. [18]. 
In order to perform further study about the nuclear radii for the selected isotopes, the proton, neutron and mass rms radii are calculated and the obtained values are presented in Tables (3), (4), and (5), respectively. It is very apparent from these tables that all calculated radii slightly increase for the sequential isotopes as the neutron number increases. The nuclear interaction between the additional neutrons and the other nucleons plays a major role in redistributing the nucleons inside nuclei, which affects the nuclear size to make it slightly larger.

Table (3)

Calculated proton rms radii (fm) for the selected Cr-isotopes.

\begin{tabular}{||c||c||c|c||c|c||c||c||c||}
\hline $\boldsymbol{A}$ & SKM & SKM* & SLY4 & SKxs25 & MSK7 & SKO & SKxta & SKxtb \\
\hline \hline 50 & 3.547 & 3.571 & 3.583 & 3.598 & 3.577 & 3.543 & 3.560 & 3.557 \\
\hline \hline 52 & 3.550 & 3.574 & 3.588 & 3.596 & 3.584 & 3.546 & 3.570 & 3.566 \\
\hline \hline 53 & 3.561 & 3.584 & 3.599 & 3.600 & 3.593 & 3.554 & 3.578 & 3.574 \\
\hline 54 & 3.571 & 3.594 & 3.610 & 3.605 & 3.601 & 3.563 & 3.585 & 3.582 \\
\hline
\end{tabular}

Table (4)

Calculated neutron rms radii (fm) for the selected Cr-isotopes.

\begin{tabular}{||c|c||c||c||c|c||c||c||c||}
\hline $\boldsymbol{A}$ & SKM & SKM* & SLY4 & SKxs25 & MSK7 & SKO & SKxta & SKxtb \\
\hline \hline 50 & 3.544 & 3.566 & 3.578 & 3.595 & 3.559 & 3.556 & 3.560 & 3.553 \\
\hline \hline 52 & 3.589 & 3.610 & 3.622 & 3.651 & 3.603 & 3.608 & 3.617 & 3.603 \\
\hline \hline 53 & 3.629 & 3.650 & 3.665 & 3.690 & 3.634 & 3.649 & 3.651 & 3.638 \\
\hline 54 & 3.667 & 3.687 & 3.705 & 3.728 & 3.665 & 3.689 & 3.687 & 3.671 \\
\hline
\end{tabular}

Table (5)

Calculated mass rms radii (fm) for the selected Cr-isotopes.

\begin{tabular}{|c|c|c|c||c|c||c|c||c|}
\hline $\boldsymbol{A}$ & SKM & SKM* & SLY4 & SKxs25 & MSK7 & SKO & SKxta & SKxtb \\
\hline \hline 50 & 3.546 & 3.568 & 3.580 & 3.596 & 3.567 & 3.550 & 3.560 & 3.555 \\
\hline \hline 52 & 3.571 & 3.594 & 3.606 & 3.626 & 3.594 & 3.580 & 3.595 & 3.586 \\
\hline \hline 53 & 3.598 & 3.620 & 3.635 & 3.649 & 3.615 & 3.606 & 3.618 & 3.609 \\
\hline 54 & 3.625 & 3.646 & 3.663 & 3.674 & 3.637 & 3.634 & 3.642 & 3.632 \\
\hline
\end{tabular}

The CDD profiles are obtained with Skyrme parameterizations that are used in this study. The obtained results are demonstrated in Figs.2(a)-2(d) and compared with the experimental data [19]. The calculated CDD for ${ }^{50} \mathrm{Cr},{ }^{52} \mathrm{Cr}$, and ${ }^{54} \mathrm{Cr}$ with $\mathrm{SKM}^{*}$ and for ${ }^{53} \mathrm{Cr}$ with MSK7, provide good agreement with the experimental data along most regions of $r$. The inset figures are plotted to achieve a better understanding of Skyrme set that succeeded in explaining the experimental data. 

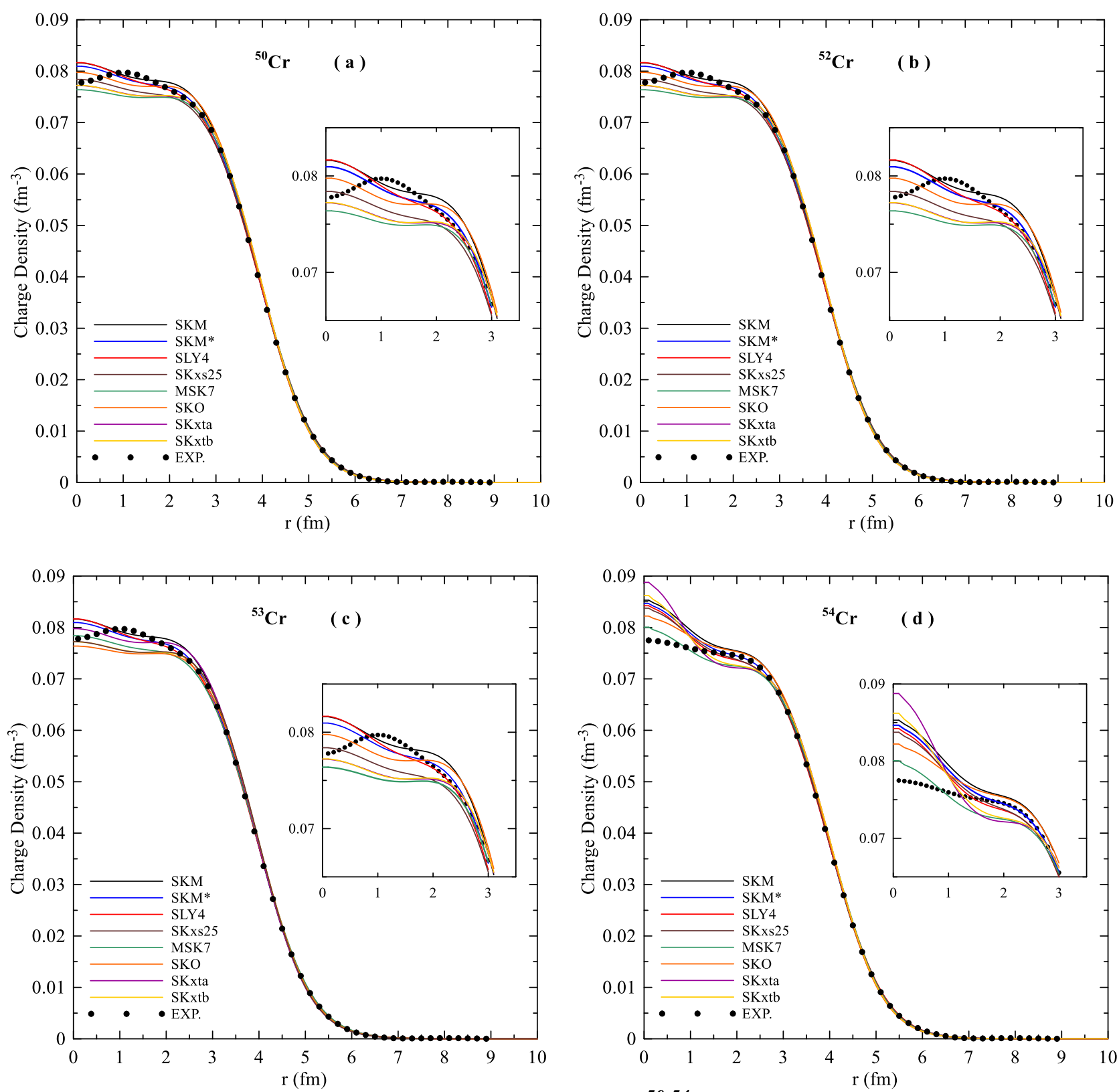

Fig.(2): Calculated charge density distributions for ${ }^{50-54} \mathrm{Cr}$ isotopes. The inset figures are embedded to clarify the agreement between the calculated CDD and experimental data. The experimental data are taken from Ref. [19].

The proton, neutron, and mass density distributions are calculated with the same Skyrme sets that were successful in providing CDD profiles completely consistent with the experimental data. These calculation are performed using SKM* for ${ }^{50} \mathrm{Cr},{ }^{52} \mathrm{Cr}$, and ${ }^{54} \mathrm{Cr}$ isotopes and MSK7 for ${ }^{53} \mathrm{Cr}$ isotope. The theoretical results are presented in Figs.3(a)3(d). The inset figures are plotted to illustrate the behavior of the density distributions at the tail regions. The Figs.3(a) and 3(b) that contain the charge and proton density distributions, show clearly the decrease and compression of the density distributions for the sequential isotopes at central and tail regions as a result of the increase of neutron number. The fall-off and the tail regions of the neutron and mass density distributions given in the Figs.3(c) and 3(d), show clearly the extension of the density distributions as a result of adding neutrons to ${ }^{50} \mathrm{Cr}$ nucleus up to ${ }^{54} \mathrm{Cr}$ nucleus. 

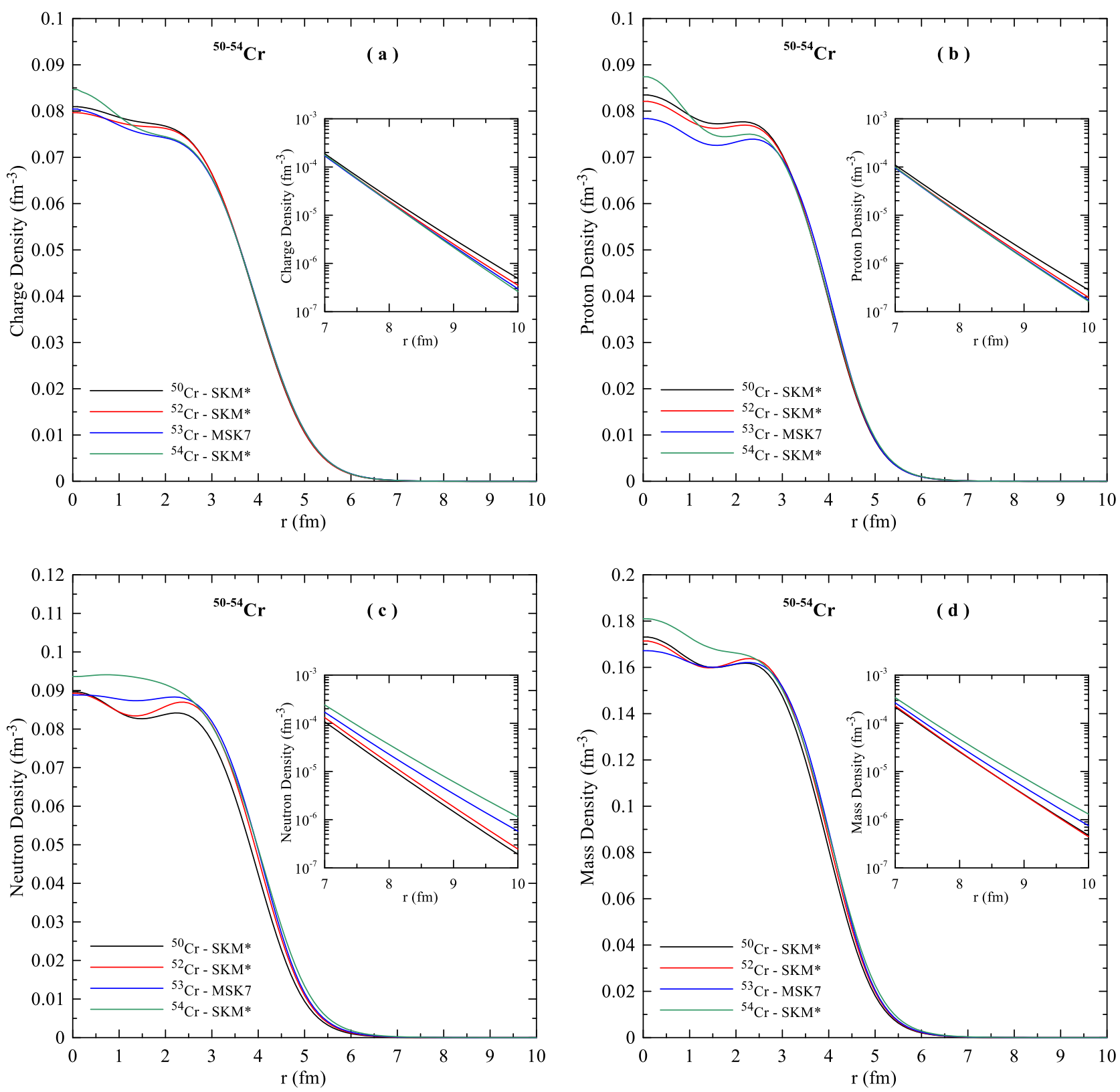

Fig.(3): Calculated density distributions for ${ }^{50-54} \mathrm{Cr}$ isotopes. The inset is in logarithmic scale. (a) charge density, (b) proton density, (c) neutron density, and (c) mass density.

The total nuclear binding energies are calculated and presented in Table (6). This Table is supported by Fig.(4) that includes a comparison between the theoretical calculated binding energy per nucleon and the experimental values [20]. The calculated binding energy of ${ }^{50} \mathrm{Cr}$ isotope with $\mathrm{SKO}$ parameterization is closer to experimental value than the other values obtained using the other Skyrme parameterizations. The calculated values for ${ }^{52} \mathrm{Cr},{ }^{53} \mathrm{Cr}$, and ${ }^{54} \mathrm{Cr}$ with $\mathrm{SKM}^{*}, \mathrm{MSK} 7$, and SKM* parameterizations, respectively, agree excellently with the corresponding experimental ones. Table (6) shows that the nuclear binding energies of the considered $\mathrm{Cr}$-isotopes increase progressively from ${ }^{50} \mathrm{Cr}$ up to ${ }^{54} \mathrm{Cr}$ with increase of neutron number. The higher values of binding energy per nucleon of ${ }^{52} \mathrm{Cr}$ isotope that are shown explicitly in Fig.(4), are attributed to the neutron magic number $(\mathrm{N}=28)$, where the nuclei having proton number $\mathrm{Z}$ or neutron number $\mathrm{N}$ or both equal to magic numbers are more stable; almost having spherical shape (small electric quadrupole moments), and higher average binding energy per nucleon in comparison with neighboring nuclei [21]. 
Table (6)

The total binding energy (MeV) for the selected Cr-isotopes.

\begin{tabular}{|l||c||c||c|c|c|c||c|c||c||}
\hline $\boldsymbol{A}$ & SKM & SKM* & SLY4 & SKxs25 & MSK7 & SKO & SKxta & SKxtb & EXP.[20] \\
\hline \hline 50 & 439.329 & 430.486 & 429.399 & 426.678 & 429.206 & 433.345 & 427.906 & 427.632 & 435.051 \\
\hline \hline 52 & 465.859 & 456.619 & 454.817 & 451.804 & 455.216 & 458.882 & 453.594 & 452.684 & 456.350 \\
\hline 53 & 474.320 & 465.244 & 462.854 & 460.949 & 464.209 & 467.404 & 462.107 & 461.161 & 464.289 \\
\hline 54 & 482.779 & 473.857 & 470.954 & 469.865 & 473.029 & 475.757 & 470.210 & 469.374 & 474.008 \\
\hline \hline
\end{tabular}

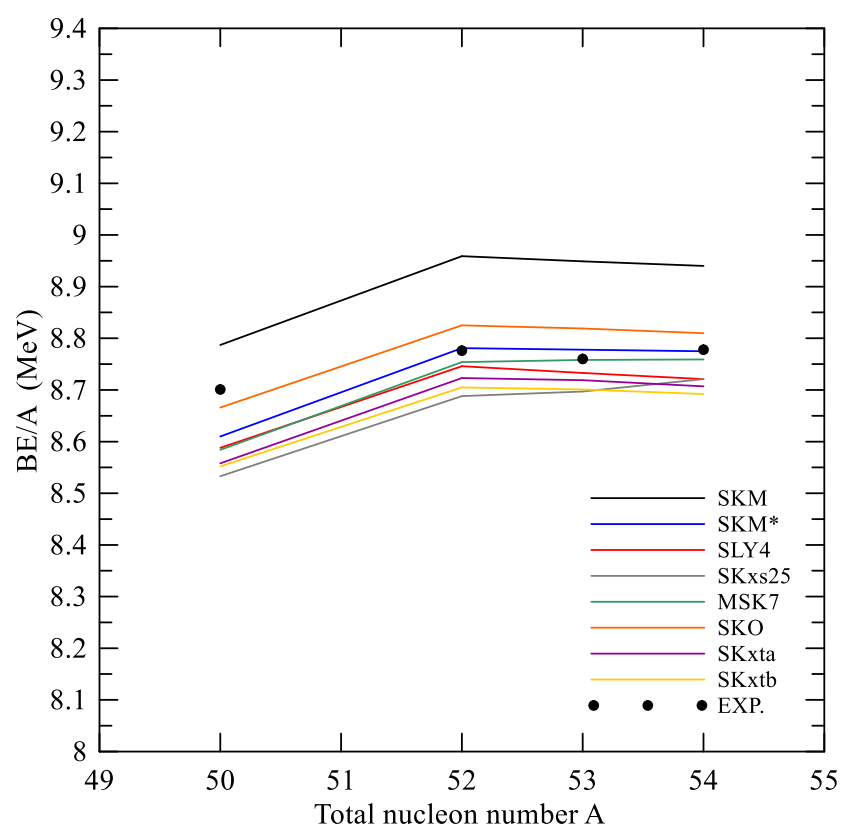

Fig.(4): Calculated binding energies per nucleon for ${ }^{50-54} \mathrm{Cr}$ isotopes compared with experimental data taken form Ref. [20].

The surplus of neutrons at the surface regions of nuclei is defined as the neutron skin thickness $(t)$ that can be deduced from the difference of the rms radii of neutrons and protons. The obtained values for all considered isotopes are listed in Table (7) and plotted in Fig.(5). The neutron skin thickness values increase with increase of the neutron number and the negative values for ${ }^{50} \mathrm{Cr}$ indicate that the proton rms radii are larger than the neutron rms radii.

Table (7)

Calculated neutron skin thickness (fm) for the selected Cr-isotopes.

\begin{tabular}{|c||c||c|c|c|c||c||c||c||}
\hline $\boldsymbol{A}$ & SKM & $\boldsymbol{S K M}^{*}$ & SLY4 & SKxs25 & MSK7 & SKO & SKxta & SKxtb \\
\hline \hline 50 & -0.004 & -0.005 & -0.005 & -0.002 & -0.018 & 0.013 & 0.000 & -0.004 \\
\hline 52 & 0.038 & 0.037 & 0.034 & 0.055 & 0.018 & 0.062 & 0.046 & 0.038 \\
\hline 53 & 0.068 & 0.066 & 0.066 & 0.090 & 0.041 & 0.094 & 0.074 & 0.063 \\
\hline 54 & 0.096 & 0.093 & 0.096 & 0.124 & 0.063 & 0.126 & 0.102 & 0.089 \\
\hline
\end{tabular}




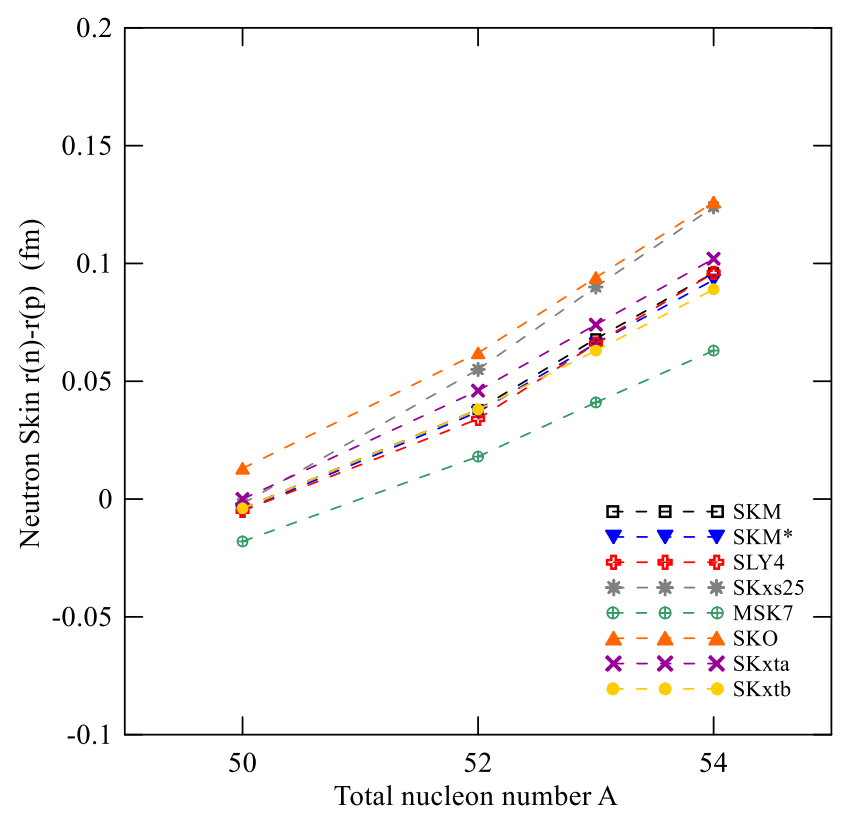

Fig.(5): The neutron skin thickness for ${ }^{50-54}$ Cr isotopes.

The elastic charge form factors are important quantities that are measured experimentally and assist to give a complete description about the charge density distributions of nuclei. The elastic charge form factors are calculated using SKM* parameterization for ${ }^{50} \mathrm{Cr},{ }^{52} \mathrm{Cr}$, and ${ }^{54} \mathrm{Cr}$ isotopes and using MSK7 parameterization for ${ }^{53} \mathrm{Cr}$ isotope, where these sets of Skyrme parameterizations have succeeded in describing the experimental data of CDD for the considered isotopes. Fig.(6), shows a comparison between the calculated (solid curves) and experimental data (hollow circles) [19]. Experimental data are explained excellently by the calculations for all isotopes for all momentum transfer regions except the small deviation noticed at high momentum transfer beyond $q \approx 2.45 \mathrm{fm}^{-1}$ for ${ }^{50} \mathrm{Cr}$ and beyond $q \approx 2.25 \mathrm{fm}^{-1}$ for ${ }^{52} \mathrm{Cr}$. This excellent agreement involved the first and the second diffraction minima that are located at $q \approx 1.1 \mathrm{fm}^{-1}$ and $q \approx 1.85 \mathrm{fm}^{-1}$ respectively.

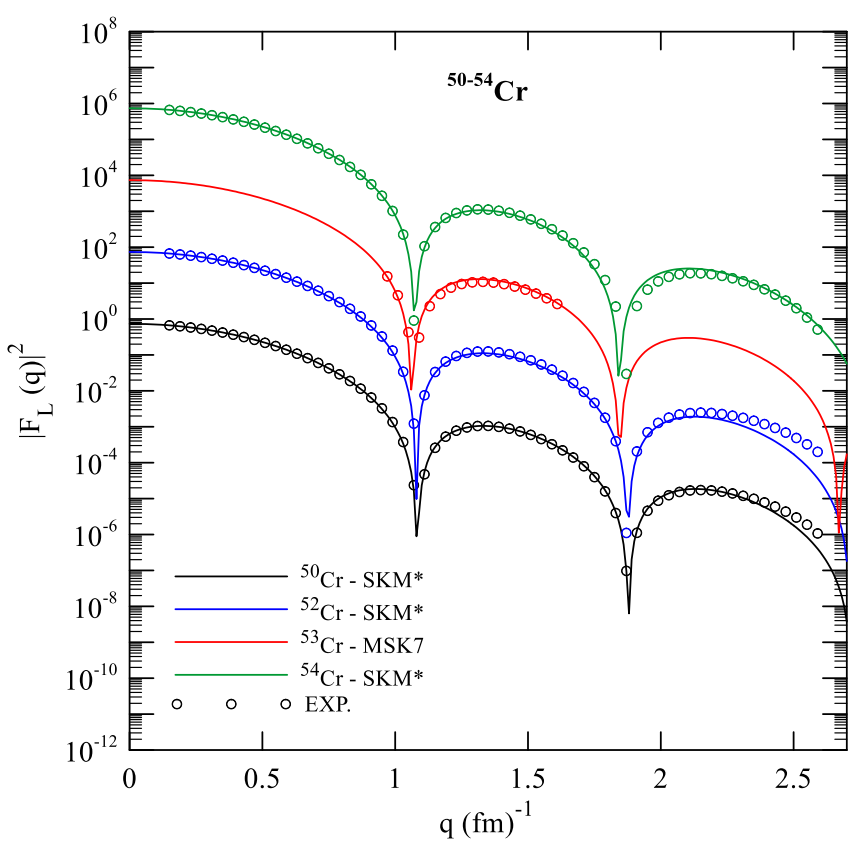

Fig.(6): The charge form factors calculated for ${ }^{50} \mathrm{Cr},{ }^{52} \mathrm{Cr}\left(\times 10^{2}\right),{ }^{53} \mathrm{Cr}\left(\times 10^{4}\right)$, and ${ }^{54} \mathrm{Cr}\left(\times 10^{6}\right)$. The experimental data are taken from Ref. [19]. 


\section{Conclusions}

From the inspection of all obtained results, one can deduce the following conclusions:

1. Our calculations confirm the ability of the SHF method to describe the nuclear structure of the spherical nuclei in its ground state.

2. The calculated CDD and the corresponding charge form factors for ${ }^{50} \mathrm{Cr},{ }^{52} \mathrm{Cr}$, and ${ }^{54} \mathrm{Cr}$ with SKM* and for ${ }^{53} \mathrm{Cr}$ with MSK7, are in excellent agreement with the experimental data.

3. The calculated binding energies for ${ }^{50} \mathrm{Cr}$, ${ }^{52} \mathrm{Cr}$, ${ }^{53} \mathrm{Cr}$, and ${ }^{54} \mathrm{Cr}$ with $\mathrm{SKO}, \mathrm{SKM}^{*}$, MSK7, and SKM* parameterizations agree excellently with the corresponding experimental ones.

4. The calculated rms radii increase progressively from ${ }^{50} \mathrm{Cr}$ up to ${ }^{54} \mathrm{Cr}$ as the neutron number increases.

5. The calculated charge rms radii for ${ }^{50} \mathrm{Cr}$, ${ }^{52} \mathrm{Cr}$, ${ }^{53} \mathrm{Cr}$, and ${ }^{54} \mathrm{Cr}$ with SLY4, SKM*, SKxta, and SLY4 are more close to the experimental data than the other values that are deduced using the other Skyrme parameterizations.

6. The charge rms radii are larger than the proton rms radii.

7. The large values of binding energy per nucleon of ${ }^{52} \mathrm{Cr}$ isotope are attributed to the magic neutron number for this isotope.

8. The neutron skin thickness increase gradually from ${ }^{50} \mathrm{Cr}$ up to ${ }^{54} \mathrm{Cr}$ as the neutron number increases.

\section{References}

[1] Alzubadi A. A., Radhi R. A. and Manie N. S., "Magnetic dipole moments, electric quadrupole moments, and electron scattering form factors of neutron-rich sd-pf cross-shell nuclei", Physical Review C 97, 064312, 2018.

[2] Xu Y., Guo H., Han Y. and Shen Q., "New Skyrme interaction parameters for a unified description of the nuclear properties", Journal of Physics G: Nuclear and Particle Physics 41, 015101, 2014.

[3] Radhi R. A., Ridha A. R. and Majeed W. Z., "Comparison between shell model and self-consistent mean field calculations for ground charge density distributions and elastic form factors of ${ }^{12} \mathrm{C}$ and ${ }^{16} \mathrm{O}$ nuclei", Indian Journal of Physics 89, 723, 2015.

[4] Bartel J., Quentin P., Brack M., Guet C. and Hakansson H. B., "Towards a Better Parametrization of Skyrme-Like Effective Forces: A Critical Study of the SkM Force", Nuclear Physics A 386, 79-100, 1982.

[5] Chabanat E., Bonche P., Haensel P., Meyer J. and Schaeffer R., "A Skyrme parametrization from subnuclear to neutron star densities Part II. Nuclei far from stabilities", Nuclear Physics A 635, 231256, 1998.

[6] Brown B. A., Shen G., Hillhouse G. C., Meng G. and Trzcinska A., "Neutron skin deduced from antiprotonic atom data", Physical Review C 76, 034305, 2007.

[7] Goriely S., Tondeur F. and Pearson J. M., "A Hartree-Fock Nuclear Mass Table", Atomic Data and Nuclear Data Tables 77, 311-381, 2001.

[8] Reinhard P. G., Dean D. J., Nazarewicz W., Dobaczewski J., Maruhn J. A. and Strayer M. R., "Shape coexistence and the effective nucleon-nucleon interaction", Physical Review C 60, 014316, 1999.

[9] Brown B. A., Duguet T., Otsuka T., Abe D., and Suzuki T., "Tensor interaction contributions to single-particle energies", Physical Review C 74, 061303, 2006.

[10] Brown B. A. and Rae W. D. M., "The shell-model code NuShellX@MSU", Nuclear Data Sheets 120, 115-118, 2014.

[11] Abdullah A. N., "Matter density distributions and elastic form factors of some two-neutron halo nuclei", PramanaJournal of Physics 89(3), 2017.

[12] Alzubadi A. A., Dakhil Z. A. and Aluboodi S. T., "Microscopic Study of Nuclear Structure for Some Zr-isotopes Using Skyrme-Hartree-Fock-Method", Journal of Nuclear and Particle Physics 4 (6), 155-163, 2014.

[13] Xiong X. Y., Pei J. C. and Chen W. J., "Extension and parametrization of highorder density dependence in Skyrme forces", Physical Review C 93, 024311, 2016.

[14] Alzubadi A. A. and Hameed D. M., "Studying the Nuclear Structure of Some Target Nuclei Used for Radiotherapy Nuclei Production by Using Skyrme- 
Hartree-Fock Method", World Journal of Nuclear Science and Technology 7, 67-83, 2017.

[15] Watanabe S., Minomo K., Shimada M., Tagami S., Kimura M., Takechi M., Fukuda M., Nishimura D., Suzuki T., Matsumoto T., et al., "Ground-state properties of neutron-rich Mg isotopes", Physical Review C 89, 044610, 2014.

[16] Sharrad F. I., Hamoudi A. K. and Radhi R. A., "Elastic Electron Scattering from Some Light Nuclei", Chinese Journal of Physics 51 (3), 452-465, 2013.

[17] Alzubadi A. A., "Investigation of nuclear structure of ${ }^{30-44} \mathrm{~S}$ isotopes using spherical and deformed Skyrme-Hartree-Fock method", Indian Journal of Physics 89 (6), 619-627, 2015.

[18] Angeli I. and Marinova K. P., "Table of experimental nuclear ground state charge radii: An update", Atomic Data and Nuclear Data Tables 99, 69-95, 2013.

[19] De Vries H., De Jager C. W. and De Vries C., "Nuclear charge-densitydistribution parameters from elastic electron scattering", Atomic Data and Nuclear Data Tables 36, 495-536, 1987.

[20] Wang M., Audi G., Wapstra A. H., Kondev F. G., MacCormick M., Xu X. and Pfeiffer B., "The Ame2012 atomic mass evaluation"; Chinese Physics C 36, 16032014, 2012.

[21] Cook N. D., "Models of the Atomic Nucleus", second edition, Springer Berlin Heidelberg, 2010. 